\title{
CakeVR: A Social Virtual Reality (VR) Tool for Co-designing Cakes
}

\author{
Yanni Mei \\ Centrum Wiskunde \& Infromatica \\ Amsterdam, Netherlands \\ yannimei951225@gmail.com \\ Huib de Ridder \\ Delft University of Technology \\ Delft, Netherlands \\ H.deRidder@tudelft.nl
}

Jie Li

Centrum Wiskunde \& Infromatica

Amsterdam, Netherlands

jie.li@cwi.nl

Pablo Cesar

Centrum Wiskunde \& Infromatica

Delft University of Technology

Amsterdam, Netherlands

P.S.Cesar@cwi.nl
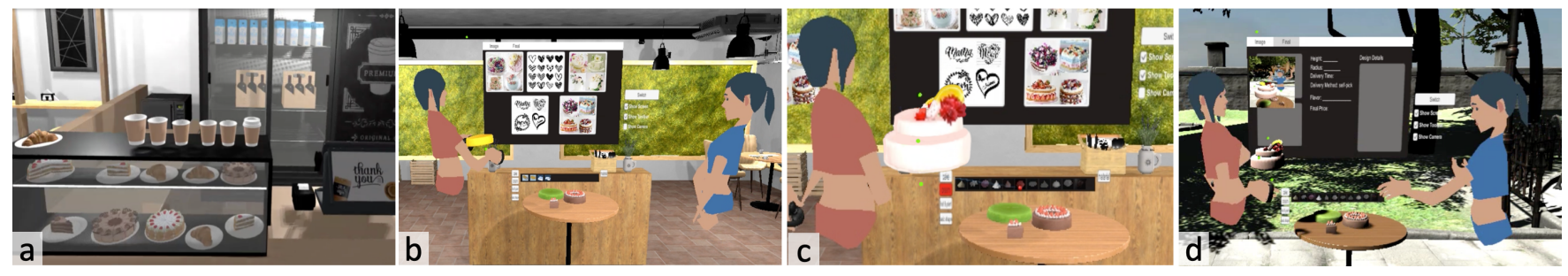

Figure 1: The CakeVR prototype: (a) both users (a pastry chef and a client) enter the virtual café; (b) upload reference cake pictures to CakeVR; (c) co-design the cake, making decisions about the size, texture, color and decorations; (d) switch the virtual café to the celebration location to check the theme fitting, take a photo of the cake, and make a final agreement.

\begin{abstract}
Cake customization services allow clients to collaboratively personalize cakes with pastry chefs. However, remote (e.g., email) and in-person co-design sessions are prone to miscommunication, due to natural restrictions in visualizing cake size, decoration, and celebration context. This paper presents the design, implementation, and expert evaluation of a social VR application (CakeVR) that allows a client to remotely co-design cakes with a pastry chef, through real-time realistic 3D visualizations. Drawing on expert semi-structured interviews (4 clients, 5 pastry chefs), we distill and incorporate 8 design requirements into our CakeVR prototype. We evaluate CakeVR with 10 experts ( 6 clients, 4 pastry chefs) using cognitive walkthroughs, and find that it supports ideation and decision making through intuitive size manipulation, color/flavor selection, decoration design, and custom celebration theme fitting. Our findings provide recommendations for enabling co-design in social VR and highlight CakeVR's potential to transform product design communication through remote interactive and immersive co-design.
\end{abstract}

Permission to make digital or hard copies of all or part of this work for personal or classroom use is granted without fee provided that copies are not made or distributed for profit or commercial advantage and that copies bear this notice and the full citation on the first page. Copyrights for components of this work owned by others than the author(s) must be honored. Abstracting with credit is permitted. To copy otherwise, or republish, to post on servers or to redistribute to lists, requires prior specific permission and/or a fee. Request permissions from permissions@acm.org.

CHI '21, May 8-13, 2021, Yokohama, Japan

(c) 2021 Copyright held by the owner/author(s). Publication rights licensed to ACM. ACM ISBN 978-1-4503-8096-6/21/05 ..\$15.00

https://doi.org/10.1145/3411764.3445503

\section{CCS CONCEPTS}

- Human-centered computing $\rightarrow$ Collaborative interaction; User centered design; Contextual design; Walkthrough evaluations; Virtual reality.

\section{KEYWORDS}

Social Virtual Reality, Co-design, Cake Design, Remote Collaboration

\section{ACM Reference Format:}

Yanni Mei, Jie Li, Huib de Ridder, and Pablo Cesar. 2021. CakeVR: A Social Virtual Reality (VR) Tool for Co-designing Cakes. In CHI Conference on Human Factors in Computing Systems (CHI '21), May 8-13, 2021, Yokohama, Japan. ACM, New York, NY, USA, 14 pages. https://doi.org/10.1145/3411764. 3445503

\section{INTRODUCTION}

Rarely is there a celebration without a cake. Apart from being an edible art, a customized cake is often a ceremonial symbol [11, 16], which is special and personal, and closely associated with social relations and emotions [28]. Customized cake services enable clients to collaboratively personalize their cake in shape, color and flavor with pastry chefs [63]. However, the customization process is not easy for both clients and chefs, which usually starts in a face-to-face meeting. Most of the follow-up communications are through text messages with the aid of reference cake pictures, which is insufficient for them to fully communicate their creative thoughts and to have a clear image of the final design [56, 78]. Cake customization requires professional skills. Based on $2 \mathrm{D}$ reference pictures and 
texts, it is not only difficult for clients to express the ideal decorations they want $[40,78]$, but also challenging for pastry chefs to immediately visualize and show the size and decorations of the cake to the clients [56]. Fig. 2 illustrates such difficulties.

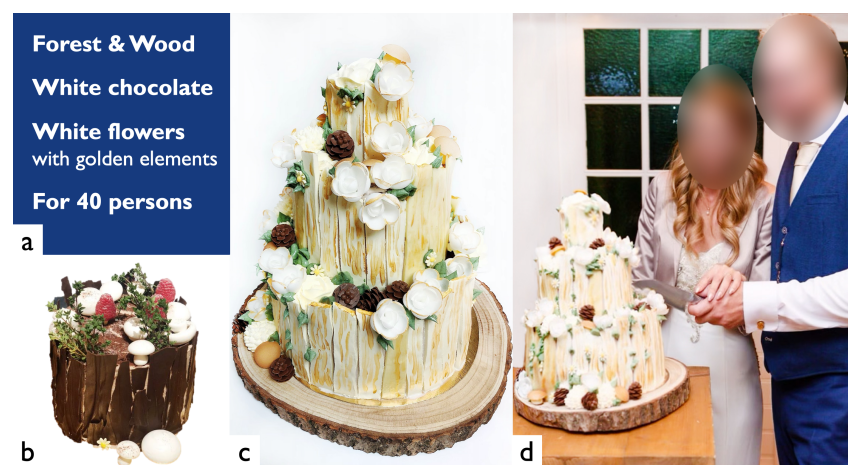

Figure 2: The difficulties in communicating the decoration and size of a customized cake: (a) design keywords from the clients, (b) a cake reference picture, (c) the final cake design in a $2 \mathrm{D}$ photo, and (d) the clients only saw the final cake at the celebration.

VR technology is developing at an unprecedented speed, which can simulate users' physical presence in a virtual environment, allow them to move around, and to interact with virtual objects. With the shifting focus from isolated experiences to a social medium, social VR has attracted a large stream of research exploring its potential for creating innovative communication approaches, supporting remote experience sharing and collaboration in diverse scientific, artistic, informational and educational domains [18, 55]. As a new remote communication medium [2], Social VR is distinguished from video conferencing tools by their capacity to portray 3D spatial information [68], to exploit users' natural behaviors, and to immerse users in the virtual world [13,32].

We posit that social VR is a promising medium to support clients to remotely co-design customized cakes with pastry chefs. Social VR allows pastry chefs and clients who are physically separated to co-present in a shared virtual space, and to assist their cake co-design by providing intuitive virtual interaction techniques and real-time 3D visualizations of virtual cakes. Both clients and chefs can instantly see the real-size 3D cake visualizations as their codesign results.

In this paper, we aim to address three research questions:

- RQ1: What is the current communication process of cake customization between clients and pastry chefs?

- RQ2: What are the design requirements for a social VR cake co-design tool (CakeVR)?

- RQ3: To what extent the design and implementation of the CakeVR prototype meet the design requirements?

Exploring cake co-design as a new social VR use case, this paper made two main contributions. First, it specifies the requirements for designing a social VR tool ( $\mathrm{CakeVR}$ ) to support remote co-design activities (Section 3). Second, it implemented the CakeVR prototype and had it evaluated by experts (i.e., pastry chefs and experienced cake customization clients) using the cognitive walkthrough method [54] (Section 4 and Section 5). The evaluation results show that CakeVR supports idea generation and decision making by allowing users to intuitively manipulate sizes, select flavors, design the decorations, and check whether the design fits the celebration theme (Section 6). We also discussed how CakeVR can be adapted to other domains, and its potential to transform product design communication through remote interactive and immersive co-design (Section 7).

\section{RELATED WORK}

The section presents related work about the co-design method, cake customization practices and social VR technology.

\subsection{Customers as co-designers}

Co-design invites customers, who are not professionally trained as designers, to actively participate in the design process and the final decision making of a product development [22], which is proved to be powerful in product innovation [57]. Co-design helps efficiently identify customers' needs [34], and enables the design outcome to achieve higher user satisfaction. Customers are transformed into codesigners, who bring their tacit and contextual knowledge to help shape design solutions [6]. Meanwhile, designers are also changed from doing design themselves to building creative environments that inspire customers to co-design with them [19, 58, 67]. Trischler et al. [67] found that the concept generated by the design team with customers achieved a much higher score in user satisfaction and novelty.

Nevertheless, co-design is confronted with challenges regarding social interaction between designers and customers. The first challenge is the trade-off between designer's authority and customer's autonomy [15]. Dahl and Moreau [14] found that autonomy (i.e., being independent, flexible and creative) and competence (i.e., having design skills or having facilitation in design) are two motivations for customers to participate in co-design. Customers often lack professional design skills to fully express design ideas [48]. However, too much guidance by designers may decline customer's feeling of autonomy. Therefore, a careful balance between designer and customers needs to be considered to ensure successful co-design experience.

The second challenge is to enable effective remote co-design, because it is not always possible to have all the co-designers physically collocated [50]. However, Sanders and Stappers [58] suggest that face-to-face interaction can support co-design to achieve the best results, because it involves complex nonverbal and tacit information exchanges (e.g., gestures [39], and aesthetic feelings [57]) that are difficult to replicate in remote communication. Thus, a new medium is needed to resemble face-to-face co-design activities, and to facilitate the competence and autonomy of co-designers.

\subsection{Cake customization}

In the late 1980s, the emerging mass customization allowed customers to configure a limited combination of features on top of a basic product to meet their individual needs [24, 47]. However, in mass customization, customers do not participate creatively in the product development, and it is often pricey if customers want to go 
further into personal choices [46]. In a recent wave of personal customization $[24,73]$, customers are invited to bring their creativity and values into the product development, and to modify the design solutions with the assistance of designers. Co-design is a helpful method to bring the customization service to a personal level [46]

Cakes are usually served on celebrations, such as weddings and birthdays. A well-decorated customized cake is always cherished as a ceremonial symbol and perceived as an edible art [16]. The demand for customized cakes has never decreased. When designing a cake, a pastry chef does not only take aesthetic and gustatory elements into account, but also considers the social meaning, the event context, and emotions related to this cake [11]. With the development of 3D food printing technologies, people can personalize their cakes in shape, color, flavor, texture and even nutrition [63]. Miyatake et al. [40] presented a 3D whipped cream printing technique for decorating cakes using a robot arm, which can dispense and stack the cream accurately according to users' drawings. Karyappa and Hashimoto [30] described an approach to accurately perform 3D printing of chocolate-based materials. In addition, many web-based or mobile applications are developed to assist product customization and provide instant 3D rendering of the customization results on screens [41]. Some bakeries provide such web-based cake customization tools. For example, Havi Proprel [49] and Baking IT [26] both offer cake configurators that provide step-by-step guidance for clients to personalize all the components of a cake (e.g., shape, filling, decoration, text and size). The customization outcome is immediately rendered and presented to customers on screens, with an automatically calculated price. However, these solutions can neither visualize the real size of the cake, nor allow customers to immersively see whether the cake design fits the celebration theme. As an emerging medium for remote communication, VR technologies have the potential to provide immersive product visualizations, real-time interactions, which can elevate the customers' customization experience in an entertaining and engaging environment [1].

\subsection{Social VR for remote communication and co-design}

Social VR is promising in approximating face-to-face interactions $[12,20,32,60]$, and has the potential to better support remote communication in terms of social presence [42]), rich non-verbal communications [71]), and immersive realistic interactions. On popular social VR platforms such as Facebook Horizon ${ }^{1}$ and AlterSpaceVR ${ }^{2}$, the facial expressions, voice, eye direction and body gestures of a user are captured and mapped to the virtual avatar of that user in real time.

Social VR has been applied in diverse domains in supporting remote multi-user collaborations. McGill et al. [38] found that, despite technical limitations of consumer VR devices (e.g., limited field-of-view), users significantly preferred the embodied social $\mathrm{VR}$ as a means of remote communication over the video-based communication. Similarly, Wang et al. [72] developed ReliveInVR, which allows multiple users to immersively relive the experience together. Reliving the experience in social VR is preferred by users

\footnotetext{
${ }^{1}$ https://www.oculus.com/facebook-horizon/

${ }^{2}$ https://altvr.com
}

to be more immersive and higher in social presence than watching the experience on screens.

In the design domain, Social VR has been used in supporting design collaborations. For instance, it provides a platform for car designers to show the 1:1 scale design details to communicate with managers who are not intimately involved in the design process [77]. Compared to desktop design tools (i.e., AutoCAD, Solidwork), VR enables immersive 3D modelling [61], and intuitive gesture-based interactions [21]. For example, VR supports 3D mid-air sketching and real-scale modeling that enables a precise understanding of the scale, proportion and spatial impacts of a design [17, 61]. Tilt Brush $^{3}$ is a well known commercial tool for 3D sketching in VR . In addition, VR enables a realistic view of the design concepts [52]. It can offer articulated visual cues (e.g., material texture) in highquality interactive 3D graphics or photo-realistic visualizations [44], which enables immediate evaluation and discussion of the design outcome [77]. Moreover, the simulated virtual space can envision possible scenarios for designers to experience how this product makes a difference in the user environment [36]. Given the advantages of VR technologies, we are specially interested in exploring a remote co-design use case using social VR.

\section{GATHERING DESIGN REQUIREMENTS}

To design a social VR tool for cake customization, semi-structured interviews were conducted with pastry chefs and clients to understand how they communicate, what types of interactions are involved from the first discussion till the agreement, and what the design requirements are for social VR tool.

\subsection{Method}

We conducted one to one semi-structured interviews [31] with five pastry chefs and four clients who had experiences in customizing cakes. Two sets of interview questions ${ }^{4}$ were designed for pastry chefs and clients, respectively. The interviews started by asking them to describe one to three memorable experiences in customizing cakes. Then, the rest of the questions guided them to talk about the events where the cakes were for, the communication methods, the challenges in communication and the final design outcomes.

All the interviews with pastry chefs were performed in-person at their bakeries. So, the chefs and the interviewer had easy access to the baking environment and tools, which could trigger discussions, and encourage chefs to tell concrete stories [10]. Two interviews with clients were performed in-person at their home, while the other two were conducted via telephone. All interviews lasted about 45 minutes, and were audio recorded. Prior to the interview, all the participants signed an informed consent and agreed that we can audio record the conversation, and use the sketches and pictures collected in the interviews. We followed a standard ethical procedure of the research institute.

\subsection{Participants}

Five pastry chefs (P1-P5, 2 males, 3 females; three aged between 30-40, and one between 40-50) and four clients (C1-C4, all females; three aged between 30-40, and one between 20-30) participated in

\footnotetext{
${ }^{3}$ https://www.tiltbrush.com

${ }^{4}$ The interview questions are available at http://tiny.cc/semi-interview-qs
} 
the interviews. All the pastry chefs are the owners of their bakeries, and are experienced in customizing cakes (7-13 years of experience). Notably, three of the clients have experiences of making cakes at home, the other one has no baking experiences. Three of the clients talked about their experiences of customizing wedding cakes, while the other one talked about a birthday cake.

\subsection{Data analysis}

With the aid of the sketches, notes and pictures collected in the interviews, the audio recordings of the interviews were transcribed. The transcripts were coded by two researchers, following an inductive open coding approach [64]. The transcripts were first coded and labeled by two researchers independently. Then, the researchers compared their coded texts. The overlapping texts were kept for later categorization. The non-overlapping texts were discussed by the two researchers, who decided together whether to keep or to discard them. Afterwards, together with a third researcher, the selected coded texts were sorted into two main categories: (1) content and process of current customization communication, and (2) design requirements for a social VR cake co-design tool.

\subsection{Results}

Based on the interviews, we identified the current communication process of cake customization and distill the design requirements for CakeVR.

3.4.1 The current communication process of cake customization. Three main phases of current cake customization communication are identified (Fig. 3) in the interview, including (1) client input, (2) ideation and negotiation, and (3) agreement. At Phase 1, a client usually starts the conversation with a pastry chef by describing the cake he or she needs from three aspects: the main features of the cake, the context where the cake will be consumed, and the emotion that the cake should convey. For explaining the main characteristics of an ideal cake, C1 said, "I wanted a big main cake in a minimalist style, with some small cakes surrounding it."). The context refers to the celebration location, the social value and the purpose of the event (P1: "The couple asked for a red cake because their wedding party would be held on a red yacht, and red is their lucky color."). The emotion aspect refers to the messages or personal stories that the cake can help convey. (P5: "This [a printed photo on a wedding cake] is the story of how the couple met."). With the aid of reference cake pictures, the three aspects of information help define the style, the size and high-level visual features (e.g., wood/forest elements) of the cake, which provide initial inspirations that lead to the Phase 2.

At Phase 2, pastry chefs help clients turn the inspirations into tangible cake designs, with professional skills and equipment. We noticed that three design strategies were often applied, namely (1) adapting from examples (reference cake pictures), (2) combining elements from examples and self designs, and (3) creating a new cake from scratch. The first design strategy allows clients to choose from existing cake examples and make limited adjustments. As P3 told us, "We offer some templates to the clients through emails or show them in our meeting. The clients can choose the color, flavor or some decorations, but there will not be a big difference". The second strategy usually combines multiple elements from reference pictures and the chefs' self-designs. (P5: "Some clients showed me a collection of cake pictures and told me, 'I like the fruit topping of this cake, and I like the flowers of that cake.' Then, we combined them into a new cake."). The third strategy is most free and creative compared to the previous two. All the elements of the cake are designed without referring to previous works. ( C2: "The pattern I drew on our wedding invitation is composed of colorful triangles. So, we thought it would be nice if the cake is also colorful and if possible also has triangles"). At this phase, clients and pastry chefs use images, sketches, sample cakes, cake dummies, in-person conversations and text messages for communication.

At Phase 3, the clients and the pastry chefs agree upon on the final design of the cake. The chefs precisely document all the design details into a formal contract, usually with a sketch or a collage of reference pictures ( $\mathrm{P} 4:$ : We collect requirements [from the clients] in a $P D F$ with the details of everything that we need to produce and deliver, and then share with them by email." $\mathrm{P} 2$ : "We include as many pictures as possible to show the expected decorations, trying to avoid any miscommunication."). The collage is more efficient, and visualizes the details and textures but fails to give an overall picture of the cake ( P2: "So, [with a photo collage], clients don't get the full visual idea of the cake, but they get the exact details they want."). The sketch offers a whole image of the cake, but is difficult to illustrate the details and textures to a photo-realistic level (P3: "It is impossible to sketch exactly the same with the real cakes."). Drawing sketches is also time-consuming and skill-demanding ( P2: "We won't draw from scratch because it was incredibly time-consuming").

3.4.2 Design requirements. To support Phase 1, it is essential to enable the pastry chef and the client to meet in the virtual space, and allow them to discuss with the aid of reference cake pictures, the celebration locations, and support the communication with natural hand gestures. As $\mathrm{C} 1$ said, "We brought the wedding invitation card to the meeting, showing our desired color of the cake to the pastry chef." $\mathrm{C} 3$ also mentioned, "I wanted the cake to fit the location of my wedding, which has a lawn and a castle in a bright summer. I searched for some images on the web to present my ideas". So, the two requirements for Phase 1 are:

R1 Allow two users represented as avatars, to enter the virtual space, and communicate with audio and gestures.

R2 Allow users to upload, grab and hold 2D reference pictures.

To support Phase 2, social VR has its unique advantage in integrating different types of media to assist design activities, and providing instant visualizations of the final design. Pastry chefs pointed out the importance of having real-time visualizations. As P3 suggested, "It is better to have an automatic visualization of the ideas, because it is difficult for clients to imagine exactly their cake just from pictures and my verbal descriptions." Similarly, C1 pointed out, "It is helpful to visualize [the cake ideas], because the ideas are often too abstract with only texts." Therefore, it is important for CakeVR to support users to perform cake co-design activities in the virtual space, including making sketches, adding decorations, resizing the cakes, and instantly see the design outcomes. There are four requirements to support Phase 2:

R3 Allow users to sketch in mid-air in the virtual space. R4 Offer pre-designed cake components (e.g., cream, fruits). 


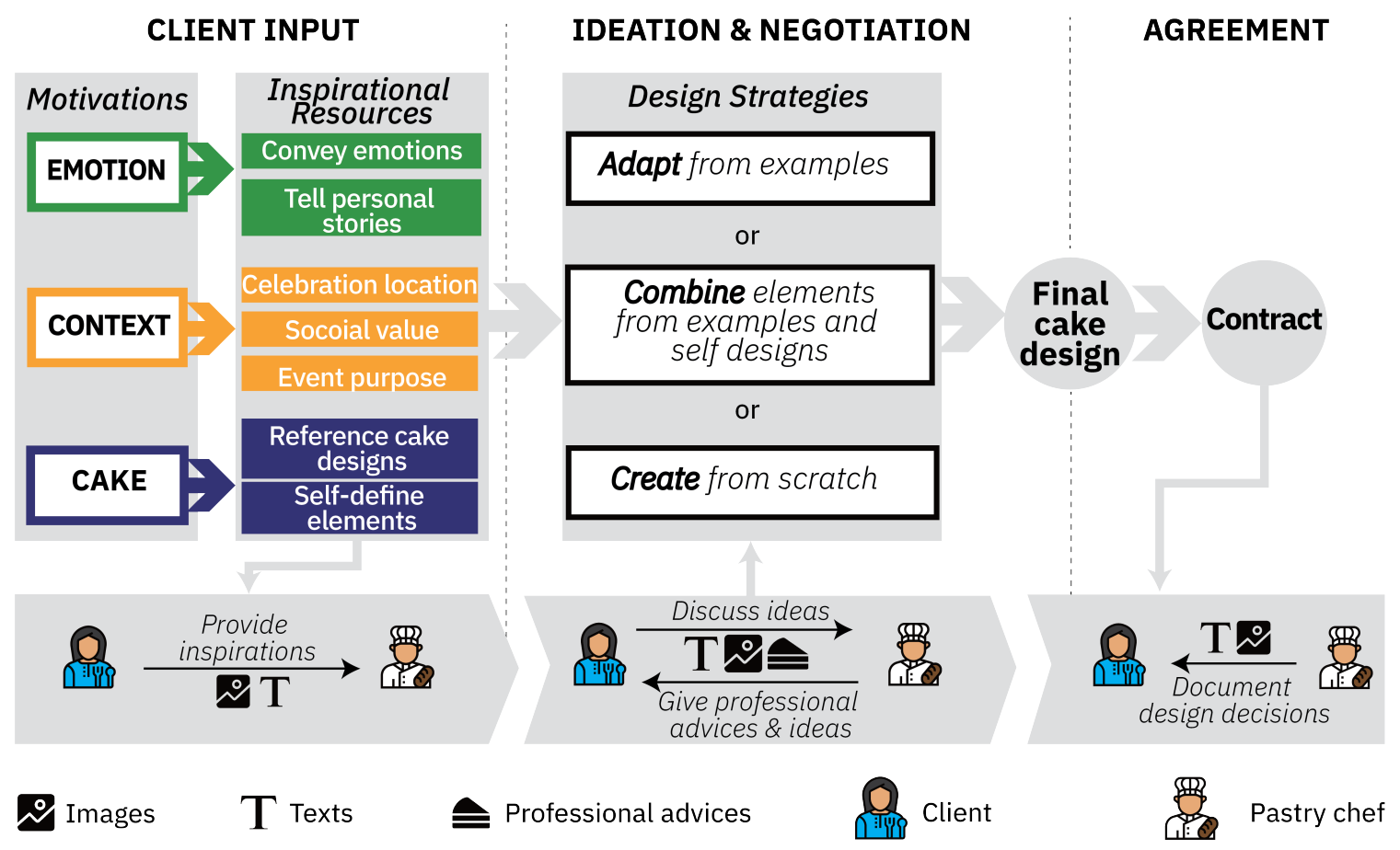

Figure 3: Three phases of current cake customization communication

R5 Allow users to see colors, textures, decorations, and real sizes of the co-design outcome in $3 \mathrm{D}$ visualizations in real time.

R6 Allow users to use intuitive gestures to interact with the interface (e.g., pointing, clicking) and to manipulate the virtual cake (e.g., resizing the cake, adding decorations).

To support Phase 3 , it is important to assist in documenting the final cake with all the design details (e.g., exact colors, texture). As P2 said, "We try to specify them [details of the cake] as much as possible in the contract." However, it happens frequently that the final design is not exactly what the clients have expected. As P1 mentioned, "The client asked for a non-bright color, but it is tricky to define 'non-bright'." C2 told us, "When I saw the cake at my wedding, the colors were different from what I had expected." CakeVR should help prevent such miscommunication. So, two requirements are derived for Phase 3:

R7 Allow users to capture photos of the cake in the virtual space. R8 Assist in documenting the design details of the virtual cake.

\section{DESIGN AND IMPLEMENTATION}

Based on the design requirements, we made a storyboard to describe the core functions and user scenarios of $\mathrm{CakeVR}$, from preparation, initial idea discussion, ideation and negotiation to confirmation (see Fig. 4). The storyboard guided the implementation of CakeVR, which is a medium-fidelity social VR prototype for one client and one pastry chef to co-design a cake in a shared virtual space. Fig. 5 illustrates the system overview of CakeVR. The virtual co-design space can switch between a virtual bakery and a celebration location. Two users who are represented as cartoon-like avatars meet at the virtual space wearing head-mounted displays (HMDs). The virtual space has a graphical interface to guide them to build a 3D virtual cake together and visualizes the cake design in real time.

The 3D cake models (e.g., Fig. 6) with textures and the predesigned cake decoration components (e.g., different shapes of cakes, cream, fruits, flowers) were made in Blender ${ }^{5}$, and then exported into Unity $3 D^{6}$ (version 2018.4.4f1). The virtual scenes including the virtual café, the garden, and the two avatars were built based on selected assets from the Unity Asset Store ${ }^{7}$. The ways in which the users interact with the virtual interface and the gestural manipulations of the virtual cake were manually coded using C\# in Unity3D. Animation Rigging, a plugin of Unity $3 D$ is applied for simulating the upper body motion of the avatars based on the spatial positions of the two hands and the head tracked by the Oculus Rifts HMD. The Oculus Rift HMD and the Oculus Touch controllers were supported by the Oculus Integration plugin. PhotonPun, a plugin of Unity, was utilized to connect two VR users and synchronize all the data (i.e., dynamic data of the 3D virtual objects, body movements of the avatars) via Internet.

Three basic gesture-based 3D manipulations were implemented in the prototype, namely moving, rotating and scaling. By pressing the grip button of the Oculus Touch controller, users can virtually grab a $3 \mathrm{D}$ object, and change its position, orientation and size. Two scaling interactions were implemented: vertical and horizontal scaling. Each 3D object has three anchor points (Fig. 7): X, Y and $Z$. User can adjust the height of the 3D objects by moving the $Z$ anchor point up or down, and change the width by dragging $X$ and $\mathrm{Y}$ in opposite directions.

\footnotetext{
$\overline{{ }^{5} \text { https://www.blender.org }}$

${ }^{6} \mathrm{https} / / /$ unity3d.com/get-unity/download

${ }^{7}$ https://assetstore.unity.com
} 


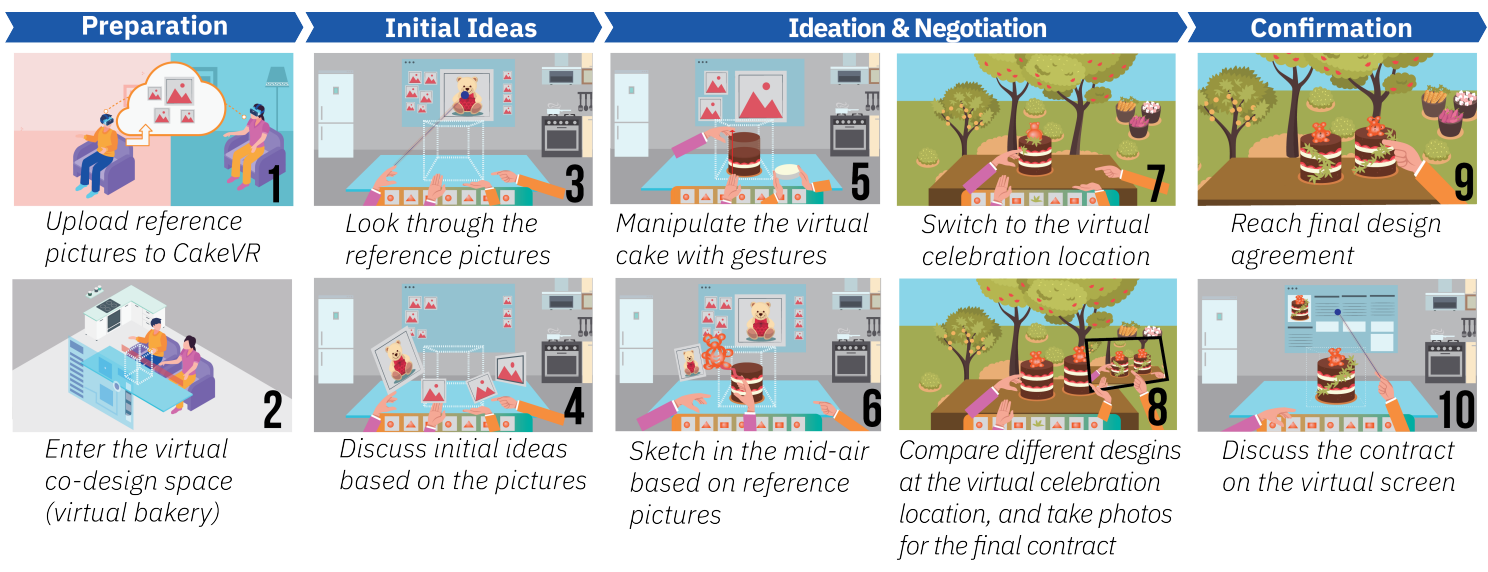

Figure 4: The storyboard of CakeVR, defining the core functions and user scenarios

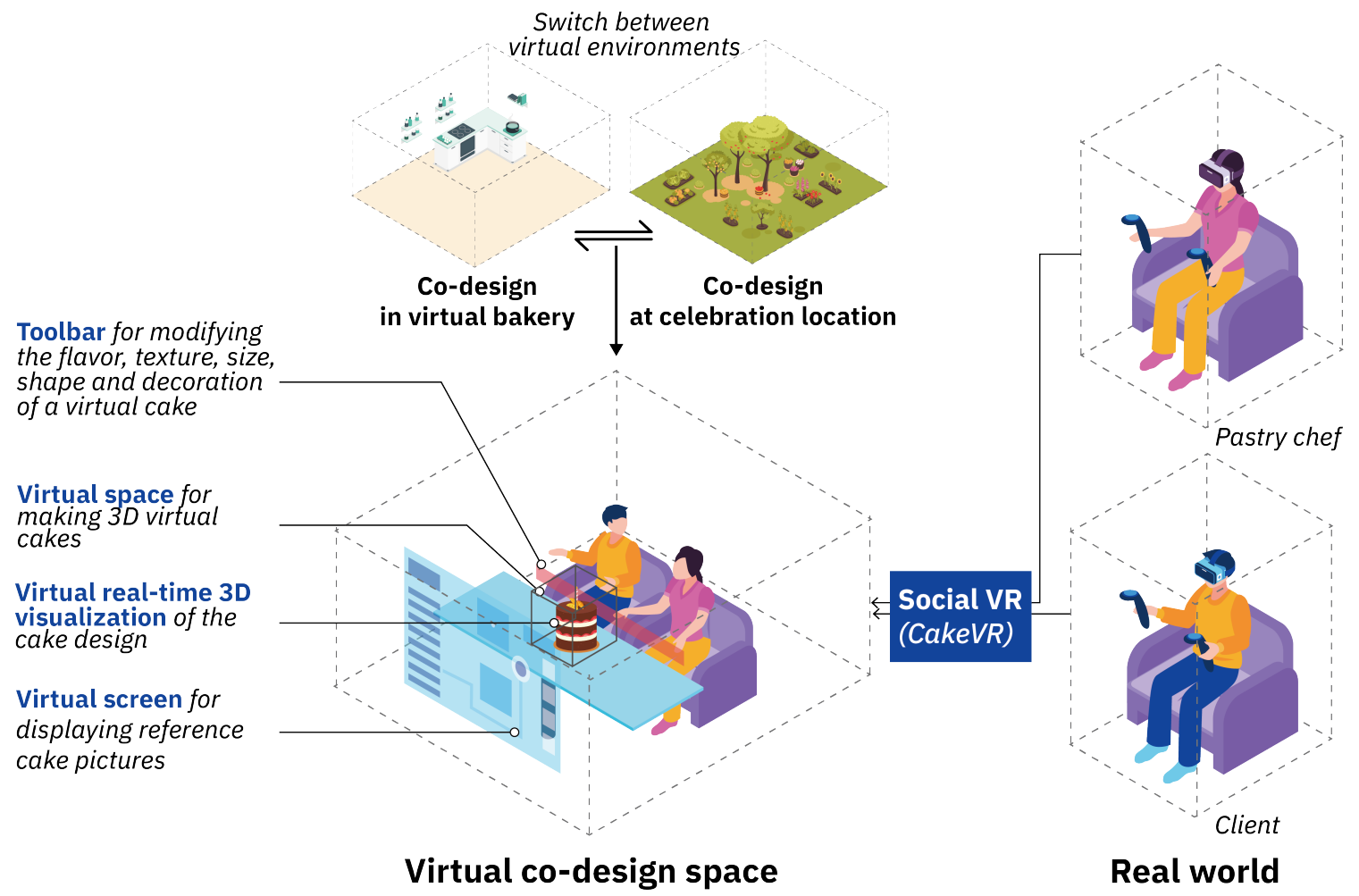

Figure 5: An overview of the CakeVR system

\section{EXPERT EVALUATION}

The goal of the expert evaluation is to invite expert users (i.e., pastry chefs and clients) who are experienced in customization cakes, to use the CakeVR prototype following a pre-defined sequence of tasks, and to check whether the design requirements are addressed in the prototype.

\subsection{Prototype fidelity and evaluation methods}

Prototype fidelity level is defined by visual refinement, breadth and depth of functionality, richness of interaction, and data model [37]. Vermeeren et al.[70] defined that prototype fidelity comprises fully functional, functional, early proof-of-concept, and non-functional. According to NASA technology readiness level (TRL) [35], our CakeVR prototype falls between TRL-3 and 4, indicating that a 


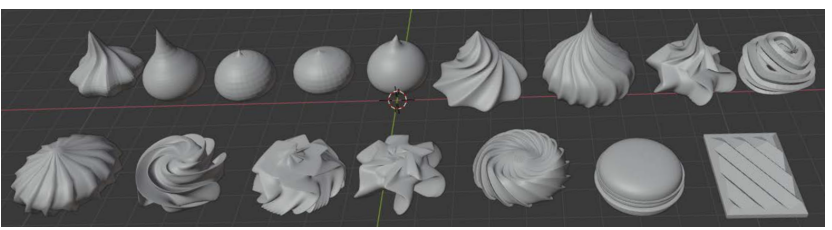

Figure 6: Pre-defined cream drops using the 3D modelling software Blender

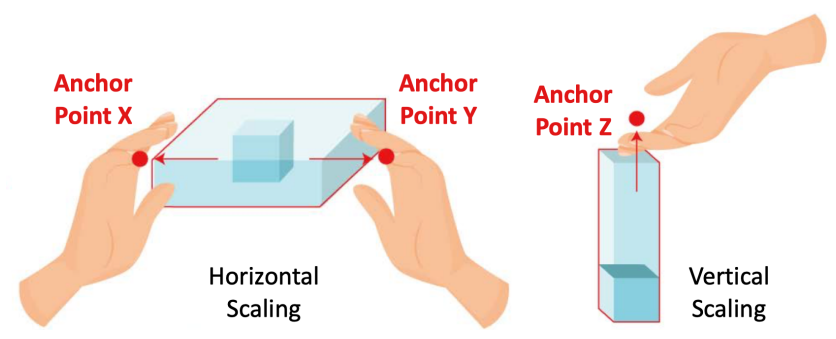

Figure 7: The horizontal and vertical scaling of the virtual object

proof-of-concept model is constructed for a laboratory test. Our CakeVR prototype can be best seen as a mid-fidelity prototype, as it is not a paper prototype, however, it lacks some functionality. It has covered the breadth of functionality (i.e., implemented all identified design requirements), but needs visual design refinement (e.g., cake fidelity), depth of functionality (e.g., precise operation), richness of interaction (e.g., facial expressions), and richness of data model (e.g., automatically calculating cake prices). For this stage, it is recommended to involve domain experts to evaluate the prototype, since their domain-specific experiences can help identify infeasible and missing functions or interactions [70].

\subsection{Method}

We adapted the cognitive walkthrough method [54] for the expert evaluation, and invited selected pastry chefs and clients as experts to test CakeVR separately. During the tests, one of the users was a researcher (experiment) who was role playing either as a pastry chef or as a client.

We chose the researcher-guided cognitive walkthrough method for two reasons:

(1) The role-playing researcher can ensure that domain experts (i.e., chefs and clients) systematically and thoroughly walk through all CakeVR functions under the same script, which enables users to have unified and comparable experiences [29].

(2) Given CakeVR's fidelity level, technical hiccups (e.g., system crash, UI freeze) due to unsupported user actions may occur, which can jeopardize the users' experience without immediate recovery. While novelty desensitization may help, it cannot fully replace the researcher guidance [70].

All the participants signed an informed consent, and gave us permissions to use the screen/audio recordings. The consent form explained the possible risks of the HMD and VR, and ensured that they can quit the test at any time. The test followed a standard ethical procedure of the research institute.

\subsection{Participants}

We invited six clients ( 3 males and 3 males, all aged between 20-30, and all were novice VR users), who are experienced cake customization customers. Five of them had one custom wedding cake experience, and the last one had more than five times custom birthday cake experiences. All of them did not participated in the previous semi-structure interviews. They are labelled as $\mathrm{C} 5-\mathrm{C} 10$, respectively. We also invited four experienced pastry chefs ( 3 females and 1 male, all aged between 30-40, three of them were novice VR users and one was an experienced VR user). One pastry chef (P1) also participated in the previous semi-structured interview. The other three were new pastry chefs, who are labelled as P6-P8, respectively. Since both clients and pastry chefs are target users of CakeVR, such mix of participants help better qualitatively evaluate the CakeVR prototype.

\subsection{Expert evaluation setup \& procedure}

The evaluation study was conducted in a room of about $25 \mathrm{~m}^{2}$, which was separated into two areas by a curtain. Each area had one computer connected with one Oculus Rifts HMD. Two users can enter CakeVR together wearing an HMD and a noise cancelling headphone. When testing with the clients, one researcher was role playing as a pastry chef. When testing with the pastry chefs, the same researcher was role playing as a client. The test followed a designed script ${ }^{8}$ to ensure that participants walked through the same four tasks: (1) exchange design ideas through reference pictures; (2) co-build cakes, (3) switch to the celebration location, take photos of the cake, and (4) finalize the contract. Prior to the test, all the participants (the clients and the pastry chefs) selected five reference cake pictures, which were uploaded to CakeVR. The test took about 1 hour, which started with a 10-minute training session for participants to learn the interaction techniques and to get familiar with the CakeVR interface. Then, the co-design session took about 15-20 minutes. The role playing researcher guided the participants to co-design cakes and to explore core functions implemented based on the design requirements. After the test in CakeVR, every participant rated CakeVR using the System Usability Scale (SUS) [7]. The SUS is a highly reliable scale intended to measure the usability and learnability of a new system $[4,8]$. The participants also participated in a 30-minute semi-structured interview ${ }^{9}$, and were asked to share their experience in terms of communication, social presence, added values and difficulties of co-designing cakes in VR, and usability of the interface.

\subsection{Data analysis}

Three types of data were collected in the expert evaluation study, namely, screen recordings of the participants' VR viewports, audio recordings of the interviews, and the SUS ratings. The screen recordings were manually annotated by two researchers to see whether participants could correctly use CakeVR, and how the co-design proceeded. For the audio-recordings, the same open coding analysis

\footnotetext{
${ }^{8}$ The script is available at http://tiny.cc/evaluation-script

${ }^{9}$ The evaluation interview questions are available at http://tiny.cc/questions-evaluation
} 
[64] was applied as we did for the previous semi-structured interviews (see Section 3.3). The results of the interview are presented in the Section 6. The SUS ratings were calculated and interpreted based on a standard template $[4,8]$.

\section{EVALUATION RESULTS}

This section presents the results of the expert evaluation, including the SUS ratings, the video annotation findings and the insights from the semi-structured interview codes.

\subsection{SUS scores}

The SUS scores given by the ten participants (6 clients: C5-C10; and 4 pastry chefs: P1, P6-P8) are presented in Fig. 8 (M=75.0, SD=13.8). As validated by Bangor et al. [4], the SUS score no fewer than 50.9 is considered as "ok", no fewer than 71.4 is "good", no fewer than 85.5 is "excellent", and no fewer than 90.9 is "best imaginable". One participant (C6) rated CakeVR as "best imaginable". Two (P1, P6) rated it as "excellent". Four (C7-C9, P7) rated it as "good". Two (C5, P8) rated it as "ok" and only one participant (C10) rated it slightly below "ok". The average SUS score of CakeVR falls between "good" and "excellent", indicating the usability and learnability of CakeVR is satisfactory.

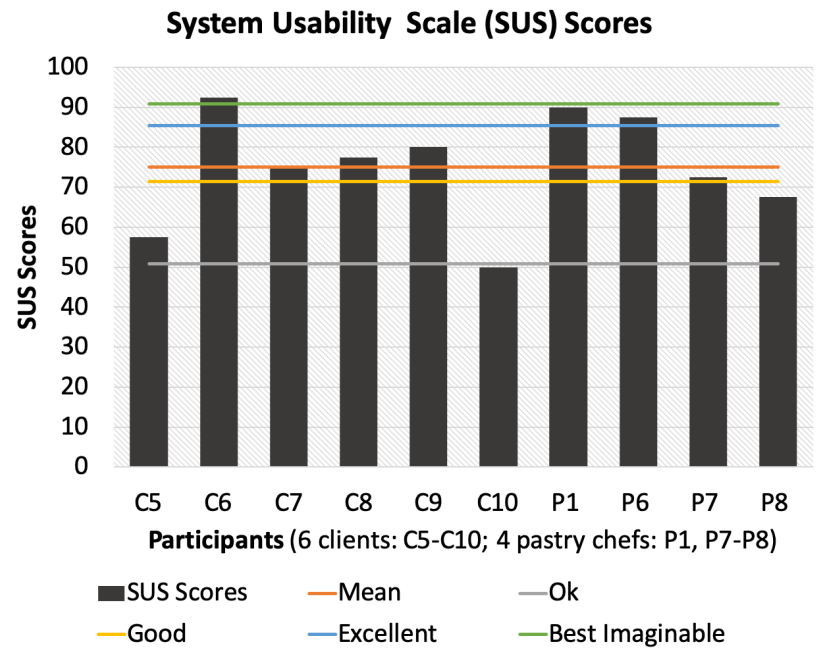

Figure 8: The SUS scores given by the ten participants in the evaluation study

\subsection{Co-design experience in CakeVR}

From the video annotations (Fig. 9), we identified two types of codesign behaviors among the six clients, which are named as the active co-designer $(\mathrm{C} 6, \mathrm{C} 9)$ and the passive co-designer $(\mathrm{C} 5, \mathrm{C} 7$, $\mathrm{C} 8, \mathrm{C} 10)$. Even though all the clients were guided to go through the same sequence of tasks, they interacted differently with the researcher who was role playing as a pastry chef. As shown in Fig. 9, the active co-designers were engaged in the cake design, spent time to try out the options on the CakeVR interface, and built the virtual cake independently. C6 and $\mathrm{C} 10$ spent noticeable amount of time on cake making (orange color). They only occasionally sought advice from the chef. In contrast, the passive co-designers often described the requirements and cake ideas to the chef. They sometimes participated in adjusting the decoration of the cake, but mostly they waited for the chef to build the virtual cake (blue color). As expected, during the test with the pastry chefs, this distinction in co-design did not exist. The pastry chefs were all actively exploring the functions of CakeVR and building virtual cakes on their own. Despite completing the same sequence of tasks, we observed that chefs $(\mathrm{N}=4)$ and clients $(\mathrm{N}=6)$ had different completion times (chefs: $M=386.25 \mathrm{~s}, \mathrm{SD}=80.87 \mathrm{~s}$; clients: $\mathrm{M}=834.17 \mathrm{~s}, \mathrm{SD}=161.00 \mathrm{~s}$ ).

\subsection{The added values of CakeVR and the fulfillment of design requirements}

From the interview transcripts of all the expert participants (the 4 pastry chefs: P1, P6-P8; and the 6 clients: C5-C10), we categorized three main added values of CakeVR, and highlighted the fulfilled design requirements.

6.3.1 Interactions in CakeVR. All participants agreed that CakeVR allowed two users to collaborate fluently with avatars, audio and gestures. There were no noticeable delays in the movement (R1 fulfilled). They all agreed that the gesture-based 3D manipulation of the virtual objects was intuitively designed. Even though most of them were novice VR users, they could quickly understand the CakeVR interface, learn to grab, move, rotate and scale the virtual objects, and sketch in the mid-air (R2, R3, R4 and R6 fulfilled). P6 said, "The interaction was straightforward and gave clear use cues." C8 claimed that, "The easy 3D manipulation of the cake certainly lowers the barrier for non-designers to perform design tasks." C6 and $\mathrm{C} 8$ both mentioned that 3D manipulation gave them a feeling of control in the design process. As C8 said, "3D manipulation enables me to express my design ideas quickly. I have a feeling of control, when I can resize the virtual cake, and show it immediately to the cake maker how large the cake should be."

6.3.2 Shared understanding in co-design. All participants agreed that CakeVR enabled them to have a shared understanding on the design process and the final decisions with their collaborators. The real-size $3 \mathrm{D}$ visualization of the co-design outcome helped users to accurately understand the style and the 3D shape of the cake (R5 fulfilled). As C9 said: "I could easily understand the cake maker because we were discussing and looking at the same image." The design interventions were also immediately shown on the virtual cake (R5 fulfilled). C7 said, "It was easy to understand 3D ideas in the virtual world because the object could be real-time edited." $\mathrm{C} 5$ also commented, "Whenever we had an idea, we could immediately edit the virtual cake and see the effects." This instant visualization aspect is also acknowledged by all the pastry chefs. As P1 said, "We both see the final cake. In reality, it might be slightly different, but you still have a quite good overview of the size, the color and some basic decorations." In addition, the natural gestures (e.g., waving hands and pointing to objects) helped users easily establish joint attention during the co-design. As C7 mentioned, "I used my two virtual hands to show my desired height of the cake, exactly like what I do in the real world." 


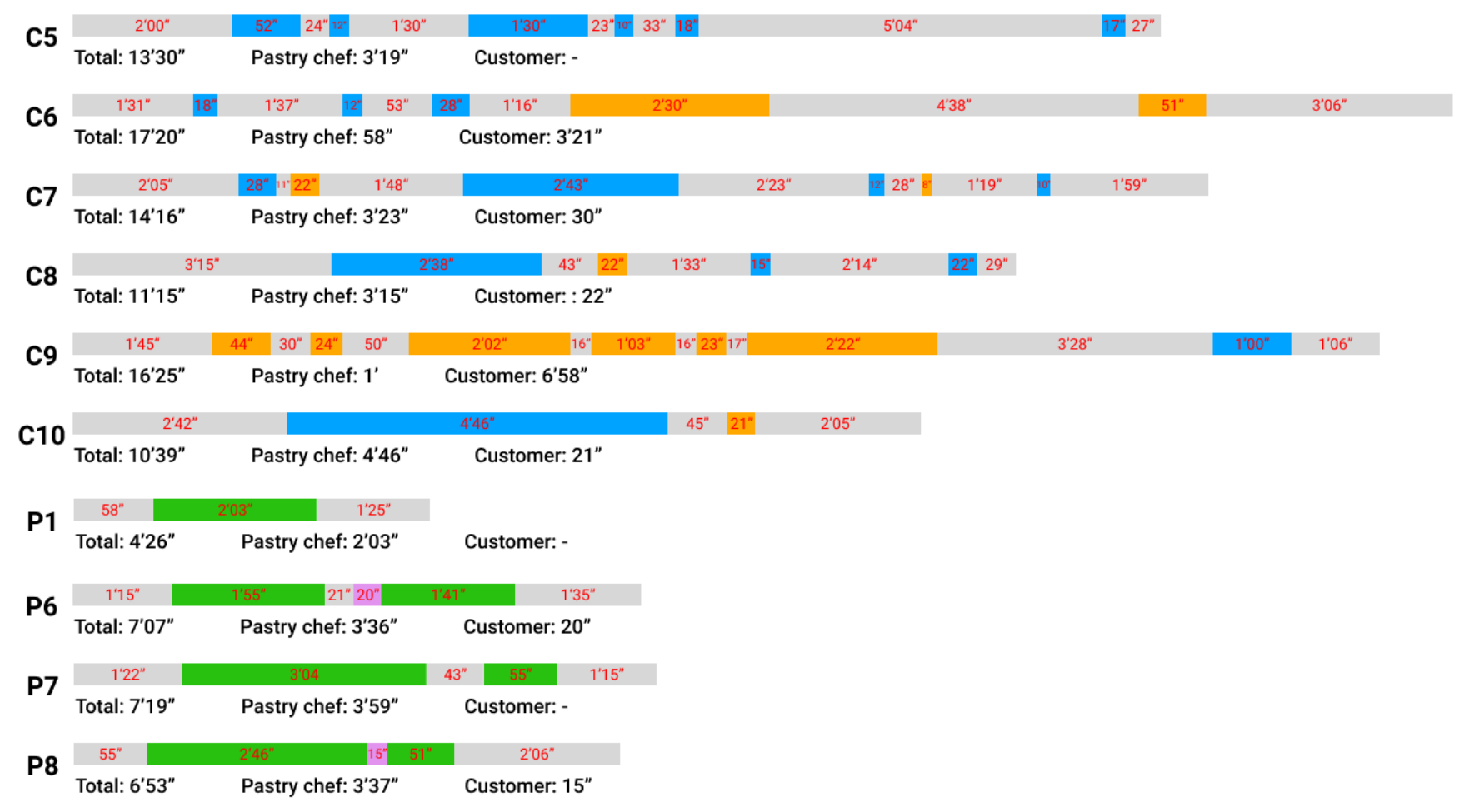

Figure 9: Video annotated time distribution of the evaluation test with the six clients (C5-C10) and the four pastry chefs (P1, P6-P8): [orange]: time the clients spent on making cakes; [blue]: time the pastry chef (role-played by the researcher) spent on making cakes; [pink] time the client (role-played by the researcher) spent on making cakes; [green] time the pastry chefs spent on making cakes; [gray]: time the clients or pastry chefs spent on consultation or exploration.

6.3.3 Efficiency in communication. P1, P7 and P8 all pointed out that CakeVR had the potential in improving the communication efficiency between clients and pastry chefs. (P1: "I can imagine if it [CakeVR] is officially in use in my bakery, it will save us a lot of time on sending multiple emails for discussing about one cake.” P8: "It [CakeVR] is quite handy, and helps me show the design of the cake immediately in front the clients. It is much easier than describing a cake in real life." P6: "It is important to have a $3 D$ scene. It is difficult to communicate about $3 D$ design on a $2 D$ screen.")

All the pastry chefs mentioned that, compared to cake customization in real life, CakeVR requires less effort in preparing materials and tools to present a 3D cake design in front of clients. (P6: "[In CakeVR], you don't need to prepare cake dummies to show the size, or prepare decoration samples. So, you can focus more on the actual design.") The efficiency in communication was also realized in the final contract support. All the pastry chefs like the idea of putting the virtual cake photos directly into the contract, which enables them to discuss and document the details of the contract immediately with the client. They would like to have further support in automatic calculation of the size and price of the cake (R7 and R8 fulfilled). As P1 said: "The final contract details take a lot of time. So, it is good to have a first discussion with the client at the end of the VR session with the virtual cake photos."

\subsection{The aspects of CakeVR that need improvement}

Apart from the added values and the fulfillment of the eight design requirements, the participants provided suggestions to further improve the CakeVR prototype.

6.4.1 Presence and social presence. All participants felt that the virtual bakery and the virtual celebration location were realistic, and they had the feeling of being there. However, they pointed out that, even though the virtual environment was realistic, they could not teleport themselves to explore it, which lowered their presence feeling (P7: "I felt it was fake because the virtual bakery looked spacious, but I could not walk around to explore it.") Four participants (C6-C9) pointed out some other factors that lowered the presence feeling, including the missing of virtual baking tools (C6) and kitchens (C9), background music and environmental noises 


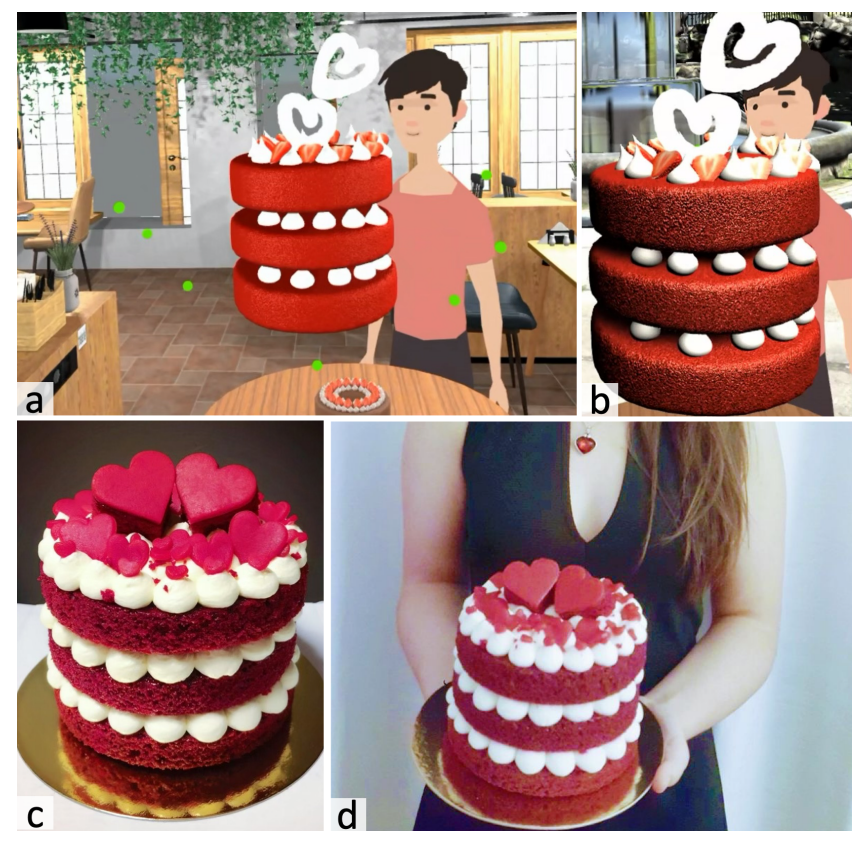

Figure 10: (a) \& (b) The virtual cake built in CakeVR by a pastry chef (P1); (c) \& (d) The real cake made by P1

(C7), and guests in the virtual café (C8). Some participants pointed out that the cartoon avatars without legs undermined the feeling of presence (C7, P6-P8). (C7: "I cannot fully feel myself in the virtual space because I don't have legs.")

Due to the lack of facial expressions, the feeling of social presence and the emotional connections between users were insufficient in CakeVR (C5, C7-C10, P1, P6-P8). Some participants mentioned that the lack of emotional connections result in the lack of engagement in the co-design activities (C5, C7, C8, C10, P7). As P7 said: "I did not really look at the avatar, because it had little reactions. I felt that I was just talking to someone via telephone." The only moment users started to pay more attention to the avatar was the photo taking moment with the co-designed virtual cakes. Participants usually got surprised to see their avatars in the selfie camera. As P6 mentioned, "Before that [taking photos of the cake with the client], I didn't really pay much attention to the client's avatar." However, six participants (C6, C9, P1, P6-P8) agreed that, not seeing the emotions from another avatar and not being able to walk around the virtual space helped them focus on the cake design work (P7: "I cannot walk around in the café, and cannot get closer to the other user's avatar, but anyway, I can focus on the task.")

6.4.2 Design resources and fidelity. Nine out of ten participants (C5-C9, P1, P6-P8) said that the the pre-designed cake components were not sufficient to support personalized co-design. These components made the cake co-design easier, but also limited the freedom and creativity. As C7 said, "I don't have the freedom to generate the $3 D$ shape I want, and I have to select between the pre-uploaded models." C8 suggested, "To better support my creativity, I would like to get access to the internet in the virtual world, to search for $3 D$ models and pictures." $\mathrm{C} 7$ and C10 would like to see 3D cake design examples in the virtual space to get inspirations, and to have a proper expectation towards the co-design outcomes (C7: "When I just entered [the virtual bakery], I had no idea what kind of cake the tool allowed me to make.") All the participants also claimed that the fidelity of the cake outcome was low, which may influence the final agreement, since clients do not really see the real cake (C6: "I can roughly imagine the overall look of the cake, but the details, like the texture, the ingredients are not realistic enough for me to imagine.")

According to the feedback of the participants, all the eight design requirements (R1-R8) are fulfilled in the CakeVR prototype. Table 1 provides a summary of future improvement suggestions from the participants.

\section{DISCUSSION}

Our findings have addressed the three research questions (RQ1RQ3). The findings of the requirement gathering interview (Section 3.4.1) describe the current communication process of cake customization between clients and pastry chefs (RQ1). The findings in Section 3.4.2 identified eight requirements based on interviews (RQ2). Our findings from our expert evaluation (Section 6 show that social VR is a suitable tool to immerse users and enable them to remotely co-design cakes (RQ3).

Even though social VR is a promising medium to support remote communication, its goal is not to completely replicate reality, but to enable possibilities to facilitate and extend existing communication channels in the physical world. However, current social VR technologies still have fundamental limitations in doing so. Here we discuss these limitations and opportunities, and provide design recommendations for social VR applications.

\subsection{Generalizability of the findings}

Our findings can generalize to a range of domains that require interaction with virtual objects, such as virtual 3D modeling or 1:1 scale prototyping for industrial design [3]; examining product details in a virtual retail store [69]; or sharing 3D medical images to manage patient expectations [9]. CakeVR further establishes the usefulness of these basic techniques (object grabbing, rotating, scaling). It additionally shows how sketching in mid-air can facilitate expression, communication, and negotiation. Our findings also generalize to virtual collaborative tasks in the new medium of social VR (cf., VRChat), and show the importance of establishing emotional connections, attention allocation, and engagement of collaboration, which have implications beyond cake co-design activities (e.g., virtual co-located learning).

\subsection{Limitations in expert evaluation}

We are aware of the limitations of the expert evaluation study. First, we did not manage to setup a real remote communication scenario. Two users who were wearing noise cancelling headphones were sharing the same room during the evaluation test. However, three pastry chefs (P1, P7, P8) mentioned that CakeVR should not only be a remote communication tool, but can also be a useful tool at the bakery, which can support on-site customization by providing instant 3D visualizations and size of the cake. Second, we did not invite clients to co-design cakes with real pastry chefs. Instead, they were interacting with a role playing researcher in the test. Since 
Table 1: The fulfilment of design requirements and future improvement suggestions

\begin{tabular}{|c|c|c|c|}
\hline $\begin{array}{l}\text { Design } \\
\text { requirements }\end{array}$ & $\begin{array}{l}\text { Fulfillment } \\
\text { (yes or no) }\end{array}$ & Suggestions for next iteration & $\begin{array}{c}\text { Participants } \\
\text { who } \\
\text { mentioned }\end{array}$ \\
\hline $\mathrm{R} 1$ & yes & $\begin{array}{l}\text { Add avatar facial expressions, enable } \\
\text { to explore virtual spaces }\end{array}$ & C5, C7-C10, P1, P6-P8 \\
\hline $\mathrm{R} 2$ & yes & $\begin{array}{l}\text { Access to the Internet within VR to } \\
\text { search for references }\end{array}$ & $\mathrm{C} 8$ \\
\hline $\mathrm{R} 3$ & yes & Sketch stably with multiple color choices & C7, P1 \\
\hline $\mathrm{R} 4$ & yes & $\begin{array}{l}\text { Offer a wide variety of components, allow } \\
\text { self-generate components }\end{array}$ & C5-C9, P1, P6-P8 \\
\hline R5 & yes & Improve the fidelity of the 3D visualization & all the 10 participants \\
\hline R6 & yes & Improve the previews of the cake components & $\mathrm{C} 7, \mathrm{C} 10$ \\
\hline R7 & yes & $\begin{array}{l}\text { Enable repositioning the cake and the avatar } \\
\text { for the photo shooting }\end{array}$ & P1, P6-P8 \\
\hline $\mathrm{R} 8$ & yes & Support automatic size and price calculation & P1, P6-P8 \\
\hline
\end{tabular}

CakeVR is at the early prototype stage, involving researchers and expert users in the evaluation and collecting rich qualitative can allow us to have a good understanding of the problem space and ensure all the core functions are addressed, which is a preparation step for a more extensive user study [57].

\subsection{Co-design in Social VR}

One common problem in co-design is that non-designer participants find it difficult to verbally describe their tacit knowledge, and to have cross-disciplinary discussions [57]. From our study, we see the potential of social VR in facilitating non-designers to express their ideas by offering ready-to-use design components and instant immersive 3D visualizations. In addition, we noticed two aspects of the 3D visualization are important: (1) showing the actual size (if the size is an important design aspect), and (2) enabling codesigners to share the view of the visualization simultaneously. These observations echo the findings by Tiainen et al. [66]: actual size and sharing views are better supported in social VR than on 2D screens and in Augmented Reality.

As addressed in Section 2.1, both autonomy and competence are important for encouraging non-designers to participate in co-design activities [14]. The two types of co-design behaviors (i.e., active and passive co-designers) identified from the expert evaluation (Section 6.2) may be related to autonomy and competence. We observed in our study that, compared to the passive co-designers, the active codesigners felt more confident, engaged and independent in the cake co-design. From the interview, we also observed two factors that may be related to the active/passive co-design behaviors, namely motivation, and emotional connection. The motivation is related to participants' personal interests and design skills, which make them more capable and more engaged in the co-design tasks. The lack of emotional connection may help participants focus on co-design. However, it may also undermine their engagement in co-design. We are interested in further researching on the two types of co-design behaviors and the ways to facilitate the competence and autonomy of users in social VR co-design.

\subsection{Design recommendations for social VR}

Based on our study, we summarized three design recommendations for developing future social VR applications.

7.4.1 Conveying emotions in social VR. We found that the lack of facial expressions reduced the emotional connection between users, which negatively influenced the engagement of some participants. We recommend that it is important to enable the virtual human representations to show facial expressions or to implement a virtual interface for users to express visually their emotions. There is an increasing number of research working on realistically representing users in the format of volumetric videos [62], removing the HMD to show the users whole face in these volumetric representations [76], and training machine learning models to estimate emotions from the images of human faces wearing an HMD [75]. Another direction is to include visual cues of emotions in social VR. For example, emoticons and emojis are effective in conveying emotions in text messages [65]. We foresee the opportunities for designing new emoticons and emojis specifically for the virtual world, where users can experience new ways to use and interact with emoticons and emojis (e.g., throw an emoji to the air). Obrist et al. [43] investigated an innovative mid-air hapitc descriptions of specific emotions (e.g., happy, sad, excited, afraid), which inspires us to think about including these haptic descriptions in the next generation of virtual input devices (e.g., hand controllers).

7.4.2 Creative virtual environment. All the participants mentioned that, although the virtual environment looks realistic, it offers limited design resources and there is a lack of serendipity to inspire them. We recommend that future social VR applications should, on one hand, provide sufficient resources (e.g., including an Internet search engine or various datasets for 3D models), and on the other hand, facilitate users to self-create virtual objects. For example, enable real-size 3D modelling or facilitate the interaction through brain-computer interfaces (BICs) [27,33]. We see potential of BICs in automatically navigating users through the virtual 
space, enabling them to interact with virtual objects through mindcontrolling, or more advancedly, generating virtual visualizations based on users' thoughts.

7.4.3 Recreating the senses in social VR. Our participants agreed that the $3 \mathrm{D}$ real-size virtual cake visualizations provide an instant overview of the co-design outcome. However, the fidelity of the virtual cake is not sufficient for them to imagine the flavor and texture of the cake. We would like to research on recreating food-related senses in social VR, such as feeling the texture of a soft chiffon cake and a fluffy velvet cake, or even simulating the taste of the flavors. With incorporating haptic devices [59, 74], the simulation of of grasping, squeezing, pressing, lifting, and stroking in VR is getting promising. The handheld controllers developed by Benko et al. [5] enable users to feel 3D surfaces, textures, and forces that match the visual rendering. Apart from the multi-sensory experiences interacting with virtual objects, recreating the senses in the virtual environment is also an interesting direction to enhance the presence of social VR users. For example, the HMD accessories developed by Ranasinghe et al. [53] provides thermal and wind stimuli to simulate real-world environmental conditions, such as ambient temperatures and wind conditions.

7.4.4 Depth of interaction and fatigue. textitCakeVR aimed to cover the breadth of interaction to ensure users can have a complete virtual cake co-design experience. However, it is interesting to consider each sub-aspect of this experience. For example, what is the impact of creating impossible cakes, and how does that push designers to think more imaginatively inside the HMD? How can specific interactions (e.g., object snapping or finalizing a scale, and by whom) create more seamless interactions? No users reported fatigue in using CakeVR, however, this needs further study for longer interaction periods, which may affect some users (e.g., older adults) [23].

\subsection{Transferability to other collaborative domains}

The audio-visual interfaces, the realistic virtual environment and the $3 \mathrm{D}$ visualizations of $\mathrm{CakeVR}$ are transferrable to other collaborative use cases, including medical and cultural heritage domains. For example, social VR can be used for remote medical consultations, enabling physicians to explain the diagnosis and its clinical impact to the patient using a 3D visualization in the virtual environment. So, the patient can share the medical decision making with the physician, and have proper expectations towards the outcomes of the treatment $[45,51]$. Social VR can enable users to relive history through interacting with the 3D mock-ups of the heritage artifacts (e.g., dressing up in a historical costumes) and talking to the virtual curators in a virtual museum [25]. Reliving the history in social VR can provide a deeper understanding of the historical accomplishments through a truly immersive, engaging experience.

\section{CONCLUSION AND FUTURE WORK}

In this paper, we designed, implemented and evaluated a social VR tool ( CakeVR) for clients to use natural gestures to co-design their dream cakes with pastry chefs in an immersive virtual bakery. Based on the eight design requirements gathered from semi-structured interviews with 5 pastry chefs and 4 clients, CakeVR was implemented for clients and chefs to co-design cakes in a virtual bakery and then switch to the celebration location to check the theme fitting. From the results of the expert evaluations (with 6 clients and 4 pastry chefs), we found that CakeVR addressed all the design requirements. The participants pointed out that $C a k e V R$ has improved the efficiency in the cake customization communication, and enhanced the shared understanding in co-design process by allowing natural gestural interactions, intuitive manipulations of $3 \mathrm{D}$ objects, and instant 3D visualizations. Our findings highlight CakeVR's potential to transform product design communication through remote interactive and immersive co-design. In our future work, we would like to further investigate social VR co-design behavior, and to recreate (food-related) senses in social VR.

\section{ACKNOWLEDGMENTS}

This research work is funded by the European Commission as part of the H2020 program, under the grant agreement 762111, "VRTogether" (http://vrtogether.eu/). The authors would like thank all the pastry chefs and clients who participated in the studies. We extend additional thanks to our colleague Abdallah El Ali (https: //abdoelali.com) who helped initiate this creative project.

\section{REFERENCES}

[1] Samar Altarteer, Charissis Vassilis, David Harrison, and Warren Chan. 2016. Product customisation: virtual reality and new opportunities for luxury brands online trading. In Proceedings of the 21st International Conference on Web3D Technology. ACM, New York, NY, USA, 173-174.

[2] Christoph Anthes, Rubén Jesús García-Hernández, Markus Wiedemann, and Dieter Kranzlmüller. 2016. State of the art of virtual reality technology. In 2016 IEEE Aerospace Conference. IEEE, Big Sky, MT, USA, 1-19.

[3] Susanna Aromaa and Kaisa Väänänen. 2016. Suitability of virtual prototypes to support human factors/ergonomics evaluation during the design. Applied ergonomics 56 (2016), 11-18.

[4] Aaron Bangor, Philip Kortum, and James Miller. 2009. Determining what individual SUS scores mean: Adding an adjective rating scale. fournal of usability studies 4, 3 (2009), 114-123.

[5] Hrvoje Benko, Christian Holz, Mike Sinclair, and Eyal Ofek. 2016. Normaltouch and texturetouch: High-fidelity $3 \mathrm{~d}$ haptic shape rendering on handheld virtual reality controllers. In Proceedings of the 29th Annual Symposium on User Interface Software and Technology. ACM, New York, NY, USA, 717-728.

[6] Sussane Bodker. 1996. Creating conditions for participation: Conflicts and resources in systems development. Human-computer interaction 11, 3 (1996), 215-236.

[7] John Brooke. 1996. SUS: a “quick and dirty'usability. CRC press, London, UK. 189 pages.

[8] John Brooke. 2013. SUS: a retrospective. Journal of usability studies 8, 2 (2013), $29-40$

[9] Elchanan Bruckheimer, Carmel Rotschild, Tamir Dagan, Gabriel Amir, Aviad Kaufman, Shaul Gelman, and Einat Birk. 2016. Computer-generated real-time digital holography: first time use in clinical medical imaging. European Heart fournal-Cardiovascular Imaging 17, 8 (2016), 845-849.

[10] Robert G Burgess. 2003. Field research: A sourcebook and field manual. Vol. 4. Routledge, London and New York.

[11] Simon R Charsley. 1992. Wedding cakes and cultural history. Taylor \& Francis, London, UK.

[12] Elizabeth F Churchill and Dave Snowdon. 1998. Collaborative virtual environments: an introductory review of issues and systems. virtual reality 3,1 (1998), 3-15.

[13] Elizabeth F Churchill, David N Snowdon, and Alan J Munro. 2012. Collaborative virtual environments: digital places and spaces for interaction. Springer Science \& Business Media, London, UK.

[14] Darren W Dahl and C Page Moreau. 2007. Thinking inside the box: Why consumers enjoy constrained creative experiences. Fournal of Marketing Research 44, 3 (2007), 357-369.

[15] Deborah Dougherty. 1992. Interpretive barriers to successful product innovation in large firms. Organization science 3, 2 (1992), 179-202.

[16] Mary Douglas. 2014. Food in the social order. Routledge, London, UK. 
[17] Tobias Drey, Jan Gugenheimer, Julian Karlbauer, Maximilian Milo, and Enrico Rukzio. 2020. VRSketchIn: Exploring the Design Space of Pen and Tablet Interaction for 3D Sketching in Virtual Reality. In Proceedings of the 2020 CHI Conference on Human Factors in Computing Systems. ACM, New York, NY, USA, 1-14.

[18] Elena Dzardanova, Vlasios Kasapakis, and Damianos Gavalas. 2018. On the effect of social context in virtual reality: An examination of the determinants of human behavior in shared immersive virtual environments. IEEE Consumer Electronics Magazine 7, 4 (2018), 44-52.

[19] Gerhard Fischer and Eric Scharff. 2000. Meta-design: design for designers. In Proceedings of the $3 r d$ conference on Designing interactive systems: processes, practices, methods, and techniques. ACM, New York, NY, USA, 396-405.

[20] Maia Garau, Mel Slater, Vinoba Vinayagamoorthy, Andrea Brogni, Anthony Steed, and M Angela Sasse. 2003. The impact of avatar realism and eye gaze control on perceived quality of communication in a shared immersive virtual environment In Proceedings of the SIGCHI conference on Human factors in computing systems. ACM, New York, NY, USA, 529-536.

[21] Sukeshini A Grandhi, Gina Joue, and Irene Mittelberg. 2011. Understanding naturalness and intuitiveness in gesture production: insights for touchless gestural interfaces. In Proceedings of the SIGCHI Conference on Human Factors in Computing Systems. ACM, New York, NY, USA, 821-824.

[22] Guido Hermans. 2014. Investigating the unexplored possibilities of digitalphysical toolkits in lay design. International fournal of Design 8, 2 (2014), 15-28.

[23] Masakazu Hirota, Hiroyuki Kanda, Takao Endo, Tomomitsu Miyoshi, Suguru Miyagawa, Yoko Hirohara, Tatsuo Yamaguchi, Makoto Saika, Takeshi Morimoto, and Takashi Fujikado. 2019. Comparison of visual fatigue caused by head-mounted display for virtual reality and two-dimensional display using objective and subjective evaluation. Ergonomics 62, 6 (2019), 759-766.

[24] S Jack Hu. 2013. Evolving paradigms of manufacturing: from mass production to mass customization and personalization. Procedia Cirp 7 (2013), 3-8.

[25] Yu-Chun Huang and Sooyeon Rosie Han. 2014. An immersive virtual reality museum via second life. In International Conference on Human-Computer Interaction. Springer, Springer, Cham, New York, NY, USA, 579-584.

[26] Baking IT. 2015. BakingIT: A Platform for Organising, Managing and Running Your Cake Business. Baking It Ltd. Retrieved September 3, 2020 from https: //www.bakingit.com

[27] Jacek Jankowski and Martin Hachet. 2013. A Survey of Interaction Techniques for Interactive 3D Environments. In Eurographics 2013 - State of the Art Reports, M. Sbert and L. Szirmay-Kalos (Eds.). The Eurographics Association, Girona, Spain, 65-93. https://doi.org/10.2312/conf/EG2013/stars/065-093

[28] Eva Johansson and Donna Berthelsen. 2014. The birthday cake: Social relations and professional practices around mealtimes with toddlers in child care. In Lived Spaces of Infant-Toddler Education and Care. Springer, New York, NY, USA, 75-88.

[29] Claire-Marie Karat, Robert Campbell, and Tarra Fiegel. 1992. Comparison of Empirical Testing and Walkthrough Methods in User Interface Evaluation. In Proceedings of the SIGCHI Conference on Human Factors in Computing Systems (Monterey, California, USA) (CHI '92). Association for Computing Machinery, New York, NY, USA, 397-404. https://doi.org/10.1145/142750.142873

[30] Rahul Karyappa and Michinao Hashimoto. 2019. chocolate-based ink threedimensional Printing (Ci3DP). Scientific reports 9, 1 (2019), 1-11.

[31] Beth L Leech. 2002. Asking questions: Techniques for semistructured interviews. PS: Political science and politics 35, 4 (2002), 665-668.

[32] Jie Li, Yiping Kong, Thomas Röggla, Francesca De Simone, Swamy Ananthanarayan, Huib de Ridder, Abdallah El Ali, and Pablo Cesar. 2019. Measuring and understanding photo sharing experiences in social Virtual Reality. In Proceedings of the ACM CHI 2019 Conference on Human Factors in Computing Systems. ACM, ACM, New York, NY, USA, 667.

[33] Fabien Lotte, Aurelien Van Langhenhove, Fabrice Lamarche, Thomas Ernest, Yann Renard, Bruno Arnaldi, and Anatole Lécuyer. 2010. Exploring large virtual environments by thoughts using a brain-computer interface based on motor imagery and high-level commands. Presence: teleoperators and virtual environments 19, 1 (2010), 54-70.

[34] Robert F Lusch and Stephen L Vargo. 2014. The service-dominant logic of marketing: Dialog, debate, and directions. Routledge, London, UK.

[35] Thuy Mai. 2012. NASA Technology Readiness Level (TRL). National Aeronautics and Space Administration. Retrieved January 4, 2020 from https://www.nasa gov/directorates/heo/scan/engineering/technology/txt_accordion $1 . h t m l$

[36] Jaques Marc, Nadira Belkacem, and Jaques Marsot. 2007. Virtual reality: A design tool for enhanced consideration of usability "validation elements". Safety science 45, 5 (2007), 589-601.

[37] Michael McCurdy, Christopher Connors, Guy Pyrzak, Bob Kanefsky, and Alonso Vera. 2006. Breaking the Fidelity Barrier: An Examination of Our Current Characterization of Prototypes and an Example of a Mixed-Fidelity Success. In Proceedings of the SIGCHI Conference on Human Factors in Computing Systems (Montréal, Québec, Canada) (CHI '06). Association for Computing Machinery, New York, NY, USA, 1233-1242. https://doi.org/10.1145/1124772.1124959

[38] Mark McGill, John H Williamson, and Stephen Brewster. 2016. Examining the role of smart TVs and VR HMDs in synchronous at-a-distance media consumption. ACM Transactions on Computer-Human Interaction (TOCHI) 23, 5 (2016), 33
[39] Albert Mehrabian. 2007. Nonverbal Communication. New Brunswick.

[40] Mako Miyatake, Aoi Watanabe, and Yoshihiro Kawahara. 2020. Interactive Cake Decoration with Whipped Cream. In Proceedings of the 2020 Multimedia on Cooking and Eating Activities Workshop. ACM, New York, NY, USA, 7-11.

[41] D Mourtzis, M Doukas, and C Vandera. 2014. Mobile apps for product customisation and design of manufacturing networks. Manufacturing Letters 2, 2 (2014), 30-34.

[42] Joschka Mütterlein, Sebastian Jelsch, and Thomas Hess. 2018. Specifics of Collaboration in Virtual Reality: How Immersion Drives the Intention to Collaborate.. In PACIS. PACIS, Yokohama, Japan, 318.

[43] Marianna Obrist, Sriram Subramanian, Elia Gatti, Benjamin Long, and Thomas Carter. 2015. Emotions mediated through mid-air haptics. In Proceedings of the 33rd Annual ACM Conference on Human Factors in Computing Systems. ACM, New York, NY, USA, 2053-2062.

[44] Sergio Orts-Escolano, Christoph Rhemann, Sean Fanello, Wayne Chang, Adarsh Kowdle, Yury Degtyarev, David Kim, Philip L Davidson, Sameh Khamis, Mingsong Dou, et al. 2016. Holoportation: Virtual 3d teleportation in real-time. In Proceedings of the 29th UIST. ACM, ACM, New York, NY, USA, 741-754.

[45] Alessandro Perin, Tommaso Francesco Galbiati, Roberta Ayadi, Enrico Gambatesa, Eleonora Francesca Orena, Nicole Irene Riker, Hagit Silberberg, Donatella Sgubin, Torstein Ragnar Meling, and Francesco DiMeco. 2020. Informed consent through 3D virtual reality: a randomized clinical trial. Acta Neurochir (Wien) April 3, 2020, online (2020), 1-8.

[46] Frank Piller, Petra Schubert, Michael Koch, and Kathrin Moesleim. 2004. From mass customization to collaborative customer codesign. In In Proceedings of the 12th European Conference on Information Systems (ECIS) 2004. ECIS, Turku, Finland, 118

[47] B Joseph Pine. 1993. Mass customization. Vol. 17. Harvard business school press Boston, Boston, Massachusetts, USA.

[48] Antti Pirinen et al. 2016. The barriers and enablers of co-design for services. International fournal of Design 10, 3 (2016), 27-42.

[49] Havi Propel. 2020. Cake Configurator. Havi Propel Product Customizer. Retrieved September 3, 2020 from https://www.havipropel.com/cake-configurator

[50] Leiying Pu. 2019. Co-design approach: a collective design method for improving working efficiency and satisfaction in user support design processes: an empirical study based on three comparison groups simulating a real-world quick start guide design process. Master's thesis. University of Twente.

[51] Andrea L Pusic, Anne F Klassen, Laura Snell, Stefan J Cano, Colleen McCarthy, Amie Scott, Yeliz Cemal, Lisa R Rubin, and Peter G Cordeiro. 2012. Measuring and managing patient expectations for breast reconstruction: impact on quality of life and patient satisfaction. Expert review of pharmacoeconomics \& outcomes research 12, 2 (2012), 149-158.

[52] Anett Racz and Gergo Zilizi. 2018. VR aided architecture and interior design. In 2018 International Conference on Advances in Computing and Communication Engineering (ICACCE). IEEE, IEEE, Paris, France, 11-16.

[53] Nimesha Ranasinghe, Pravar Jain, Shienny Karwita, David Tolley, and Ellen YiLuen Do. 2017. Ambiotherm: enhancing sense of presence in virtual reality by simulating real-world environmental conditions. In Proceedings of the $2017 \mathrm{CHI}$ Conference on Human Factors in Computing Systems. ACM, New York, NY, USA, 1731-1742.

[54] John Rieman, Marita Franzke, and David Redmiles. 1995. Usability evaluation with the cognitive walkthrough. In Conference companion on Human factors in computing systems. ACM, New York, NY, USA, 387-388.

[55] Jose Luis Rubio-Tamayo, Manuel Gertrudix Barrio, and Francisco García García. 2017. Immersive environments and virtual reality: Systematic review and advances in communication, interaction and simulation. Multimodal Technologies and Interaction 1, 4 (2017), 21.

[56] Bhawna Sachdeva. 2015. How to Prepare and Conduct Cake Consultation. Baking It Ltd. Retrieved September 1, 2020 from https://blog.bakingit.com/how-toprepare-and-conduct-cake-consultation

[57] Elizabeth B-N Sanders and Pieter Jan Stappers. 2008. Co-creation and the new landscapes of design. Co-design 4, 1 (2008), 5-18.

[58] Liz Sanders. 2012. Convivial Toolbox: Generative Research for the Front End of Design. BIS Publisher, Amsterdam, The Netherlands.

[59] Samuel B Schorr and Allison M Okamura. 2017. Fingertip tactile devices for virtual object manipulation and exploration. In Proceedings of the 2017 CHI Conference on Human Factors in Computing Systems. ACM, New York, NY, USA, 3115-3119.

[60] Harrison Jesse Smith and Michael Neff. 2018. Communication behavior in embodied virtual reality. In Proceedings of the 2018 CHI Conference on Human Factors in Computing Systems. ACM, New York, NY, USA, 1-12.

[61] Rainer Stark, Johann Habakuk Israel, and Thomas Wöhler. 2010. Towards hybrid modelling environments-Merging desktop-CAD and virtual reality-technologies. CIRP annals 59, 1 (2010), 179-182.

[62] Shishir Subramanyam, Jie Li, Irene Viola, and Pablo Cesar. 2020. Comparing the Quality of Highly Realistic Digital Humans in 3DoF and 6DoF: A Volumetric Video Case Study. In 2020 IEEE Conference on Virtual Reality and 3D User Interfaces (VR). IEEE, IEEE, Atlanta, Georgia, USA, 127-136. 
[63] Jie Sun, Zhuo Peng, Weibiao Zhou, Jerry YH Fuh, Geok Soon Hong, and Annette Chiu. 2015. A review on 3D printing for customized food fabrication. Procedia Manufacturing 1 (2015), 308-319.

[64] David R Thomas. 2006. A general inductive approach for analyzing qualitative evaluation data. American journal of evaluation 27, 2 (2006), 237-246.

[65] Dominic Thompson and Ruth Filik. 2016. Sarcasm in written communication Emoticons are efficient markers of intention. Journal of Computer-Mediated Communication 21, 2 (2016), 105-120.

[66] Tarja Tiainen and Tiina Jouppila. 2019. Use of Virtual Environment and Virtual Prototypes in Co-Design: The Case of Hospital Design. Computers 8, 2 (2019) 44.

[67] Jakob Trischler, Simon J Pervan, Stephen J Kelly, and Don R Scott. 2018. The value of codesign: The effect of customer involvement in service design teams. Journal of Service Research 21, 1 (2018), 75-100.

[68] Jose E Venson, Jean Berni, Carlos S Maia, A Marques da Silva, Marcos d'Ornelas, and Anderson Maciel. 2016. Medical imaging VR: can immersive 3D aid in diagnosis?. In Proceedings of the 22nd ACM VRST. ACM, ACM, New York, NY, USA, 349-350.

[69] Adrien Verhulst, Jean-Marie Normand, Cindy Lombart, and Guillaume Moreau. 2017. A study on the use of an immersive virtual reality store to investigate consumer perceptions and purchase behavior toward non-standard fruits and vegetables. In 2017 IEEE Virtual Reality (VR). IEEE, IEEE, Los Angeles, California, USA, 55-63.

[70] Arnold P. O. S. Vermeeren, Effie Lai-Chong Law, Virpi Roto, Marianna Obrist, Jettie Hoonhout, and Kaisa Väänänen-Vainio-Mattila. 2010. User Experience Evaluation Methods: Current State and Development Needs. In Proceedings of the 6th Nordic Conference on Human-Computer Interaction: Extending Boundaries (Reykjavik, Iceland) (NordiCHI '10). Association for Computing Machinery, New
York, NY, USA, 521-530. https://doi.org/10.1145/1868914.1868973

[71] Vinoba Vinayagamoorthy, Maxine Glancy, Christoph Ziegler, and Richard Schäffer. 2019. Personalising the TV Experience using Augmented Reality: An Exploratory Study on Delivering Synchronised Sign Language Interpretation. In Proceedings of the ACM CHI 2019 Conference on Human Factors in Computing Systems. ACM, ACM, New York, NY, USA, 532.

[72] Cheng Yao Wang, Mose Sakashita, Upol Ehsan, Jingjin Li, and Andrea Stevenson Won. 2020. Again, Together: Socially Reliving Virtual Reality Experiences When Separated. In Proceedings of the 2020 CHI Conference on Human Factors in Computing Systems. ACM, New York, NY, USA, 1-12.

[73] Yi Wang, Hai-Shu Ma, Jing-Hui Yang, and Ke-Sheng Wang. 2017. Industry 4.0: a way from mass customization to mass personalization production. Advances in Manufacturing 5, 4 (2017), 311-320.

[74] Tong Xue, Jie Li, Guo Chen, and Pablo Cesar. 2020. A Social VR Clinic for Knee Arthritis Patients with Haptics. In Proceedings of the 2020 ACM IMX Conference on Interactive Media Experiences. ACM IMX 2020, Barcelona, Spain, $1==5$.

[75] Hwanmoo Yong, Jisuk Lee, and Jongeun Choi. 2019. Emotion Recognition in Gamers Wearing Head-mounted Display. In 2019 IEEE Conference on Virtual Reality and 3D User Interfaces (VR). IEEE, IEEE, Osaka, Japan, 1251-1252.

[76] Yajie Zhao, Qingguo Xu, Weikai Chen, Chao Du, Jun Xing, Xinyu Huang, and Ruigang Yang. 2019. Mask-off: Synthesizing face images in the presence of head-mounted displays. In 2019 IEEE Conference on Virtual Reality and 3D User Interfaces (VR). IEEE, IEEE, Osaka, Japan, 267-276.

[77] Peter Zimmermann. 2008. Virtual Reality Aided Design. A survey of the use of VR in automotive industry. Springer Netherlands, Dordrecht, 277-296.

[78] Paul Zipkin. 2001. The limits of mass customization. MIT Sloan management review 42, 3 (2001), 81. 